\title{
The Modulation of Stimulus Familiarity on the Repetition Effect in Duration Judgment
}

\author{
Lina Jia ${ }^{1}$, Can Deng ${ }^{1}$, Lili Wang ${ }^{2}$, Xuelian Zang ${ }^{3 *}$ and Xiaocheng Wang ${ }^{1 *}$ \\ ${ }^{1}$ Department of Education, School of Humanities, Jiangnan University, Wuxi, China, ${ }^{2}$ Department of Psychology, Huaiyin \\ Normal University, Huai'an, China, ${ }^{3}$ School of Education, Institutes of Psychological Sciences, Hangzhou Normal University, \\ Hangzhou, China
}

OPEN ACCESS

Edited by:

Gianluca Campana,

University of Padua, Italy

Reviewed by:

Giovanna Mioni,

University of Padua, Italy

Alberto Dominguez,

University of La Laguna, Spain

*Correspondence: Xuelian Zang zangxuelian@gmail.com Xiaocheng Wang cheng.wx@jjiangnan.edu.cn

Specialty section: This article was submitted to

Perception Science,

a section of the journal

Frontiers in Psychology

Received: 30 December 2019

Accepted: 07 May 2020

Published: 12 June 2020

Citation:

Jia L, Deng C, Wang L, Zang X and Wang $X$ (2020) The Modulation of Stimulus Familiarity on

the Repetition Effect in Duration Judgment. Front. Psychol. 11:1181.

doi: 10.3389/fpsyg.2020.01181
The repetition of a stimulus often produces a shorter subjective duration than does the presentation of a novel item. To test whether familiarity mediates the repetition compression effect, the present study compared the influence of repeated words and pseudowords on apparent duration, using a duration discrimination task. We found a similar magnitude of temporal compression for the repeated-word and repeated-pseudoword conditions. When introducing a further experiment with two new conditions in which the standard-comparison pair shared a character at the first or second constituent position, we observed a shorter subjective duration for whole word (or whole pseudoword) repetition compared with the remaining conditions (i.e., first-character repetition, second-character repetition, and novel baseline). However, temporal compression for the first- and second-character repetitions was observed only for pseudowords but not for words. Our findings indicate that familiarity modulates the perception of duration in constituent character repetition. The results are discussed on the basis of the predictive coding theory.

Keywords: duration judgment, repetition compression, constituent repetition, Chinese, predictive coding

\section{INTRODUCTION}

Prior experience with a given stimulus ultimately affects how it is perceived, usually resulting in faster reaction times and/or better accuracy to judge the repeated items (Tenpenny, 1995). This effect is observed with various visual and auditory stimuli ranging from simple to complex forms. Importantly, and central to this study is that prior experience has also been shown to influence a range of temporal judgment tasks, where the presentation of a stimulus is usually perceived as shorter in duration when it is a repeat of the preceding item (Matthews, 2011; Birngruber et al., 2015; Matthews and Gheorghiu, 2016). Despite the fact that the foundation for the repetition effect on time duration still remains a subject of debate, three main accounts have been put forward. These can be subdivided into two main theoretical approaches (Matthews et al., 2014; Matthews and Gheorghiu, 2016; Ulrich and Bausenhart, 2019). The first theoretical approach includes the attentional and arousal accounts, both derived from the pacemaker-accumulator framework, which assumes an inherent timing process, referred to as the classical internal clock model (Treisman, 1963; Gibbon, 1991; Wearden, 1992; Zakay and Block, 1997). In this model, a switch connects the pacemaker and the accumulator by switching on and off to count the pulses generated by the pacemaker. Under this framework, novel stimuli are more arousing and/or capture more 
attention, thus accelerating the pacemaker, which generates more temporal pulses. As a result, the total number of pulses (and their respective intervals) counted by the accumulator leads to an overestimation of perceived duration (Tse et al., 2004; Ulrich et al., 2006; Ciria et al., 2019). To illustrate, using the oddball paradigm, Tse et al. (2004) found that an oddball stimulus (e.g., expanding disk) embedded in a train of repeated stimuli (e.g., black stationary disk) was perceived as longer than the repeated ones. The authors concluded that the repetition effect depended on the orientation of attention in response to the relative saliency of the stimuli.

The second theoretical approach is based on neurophysiological findings where reduced neural activity was observed in response to repeated stimuli, a phenomenon referred to as repetition suppression (Desimone, 1996; Henson and Rugg, 2003; Grill-Spector et al., 2006; Eagleman and Pariyadath, 2009). This effect leads to a shortened apparent duration of repeated stimuli compared to novel ones (Birngruber et al., 2015; Cai et al., 2015). Accordingly, the repetition suppression could be due to a low-sensory adaptation or a more efficient representation (Desimone, 1996; De Baene and Vogels, 2010). The latter relates to the framework of predictive coding (Matthews et al., 2014) wherein prior exposure produces a prediction that the same stimulus will recur. In order to perform efficient coding, the brain does the minimum necessary to process repeated stimuli and only thoroughly processes the stimulus deviant from the implicit expectation, a process referred to as surprising prediction errors (Rao and Ballard, 1999; Friston, 2005; Eagleman and Pariyadath, 2009). In other words, neural responses are suppressed for the predicted signals (repeated stimuli) and activated or even enhanced for the surprising stimuli (oddball/novel stimuli). The view of predictive coding was supported by a large number of studies (Matthews, 2011; Schindel et al., 2011; Pariyadath and Eagleman, 2012; Birngruber et al., 2015; Jia and Shi, 2017; Saurels et al., 2019). In this framework, the size of the repetition effect should be dependent on the similarity between the predicted and actual signals. For instance, the number of changes (e.g., varying the angles of lines) between the oddball and standard stimuli has been shown to modulate the magnitude of the oddball effect (Schindel et al., 2011; Pariyadath and Eagleman, 2012). However, more recent studies found that the repetition duration compression was reduced or even reversed when repeats were frequent compared with when repeats were rare across blocks, indicating an interaction between low-sensory adaptation and high-level expectation (Matthews, 2015; Skylark and Gheorghiu, 2017).

An additional branch of research has examined the influence of familiar (e.g., word) or unfamiliar stimuli (e.g., pseudowords) on the repetition effect over duration perception. It has been shown that both categories are prone to duration compression in relation to novel items (Matthews, 2011, 2015; Birngruber et al., 2015; Cai et al., 2015; Jia and Shi, 2017). As an example, in a duration discrimination task, in which the participants had to respond whether the presentation time of the comparison stimulus was shorter or longer than that of the baseline, Birngruber et al. (2015) found that the presentation of repeated unfamiliar pseudowords was judged as shorter in duration than that of a novel baseline stimulus. Along with a similar method, namely, two-interval paradigm, Jia and Shi (2017) showed that participants tended to underestimate the apparent duration of the repetition of familiar stimuli (i.e., Chinese character).

Although the aforementioned studies demonstrated that the repetition of familiar or unfamiliar stimuli produces duration compression relative to novel stimuli (Matthews, 2011; Birngruber et al., 2015; Cai et al., 2015; Jia and Shi, 2017), it is vital to highlight that there has been little evidence of how the familiarity (i.e., words vs. pseudowords in the same study) influences the size of the repetition duration effect. Specifically, the question is to what extent familiarity interacts with the repetition duration effect. To date, a few studies employing lexical decision and identification tasks have shown that the effect caused by the repetition of familiar stimuli was more pronounced than that of unfamiliar stimuli (Henson et al., 2000; Fiebach et al., 2005; Orfanidou et al., 2006). Moreover, neuroimaging studies revealed that as neural responses to repeated-familiar stimuli (e.g., famous faces and words) decreased, the neural responses to repeated-unfamiliar stimuli (e.g., non-famous faces and pseudowords) increased (Henson et al., 2000; Fiebach et al., 2005). Nevertheless, it is worth noting that the tasks mentioned above involved access to semantic representations in the high-level hierarchy. These tasks differ from duration judgment tasks in the sense that the latter do not require semantic-related judgment but rather judge the speed of time passage of a stimulus. For this reason, it is essential to use duration judgment tasks to investigate whether stimulus familiarity mediates the repetition duration effect.

A further point fundamental to the current study concerns the interaction of stimulus familiarity with the repetition duration effect using constituent vs. whole stimulus representation. This is important because the extant line of investigation has mainly focused on the effects of whole-stimulus repetition (e.g., picture, simple symbol, and character). It is important to highlight that these studies demonstrated the repetition duration compression (Matthews and Gheorghiu, 2016) and explained the phenomenon with bases on the predictive coding account (Schindel et al., 2011; Pariyadath and Eagleman, 2012; Jia and Shi, 2017; Saurels et al., 2019). Under this account, the magnitudes of the repetition effect reflect the discrepancy between the predicted and actual items in terms of their physical characteristics. On this note, it is theoretically critical to compare the effects of whole repetition and constituent repetition in duration perception. In particular, the investigation of constituent repetition can bring to the fore how it interacts with stimulus familiarity. What is more, until now, most empirical evidence for the constituent repetition effect comes from studies using various non-temporal tasks (Zhou et al., 1999; Tsang et al., 2014; Wu et al., 2016), such as the study by Zhou et al. (1999), which found the repetition effect in an identification task even when the standard and comparison words shared only one constituent character (e.g., “华丽-magnificent” vs. “华贵-luxurious”). In the present study, however, we ask the question as to whether familiarity mediates the effect of constituent repetition in a duration judgment task. 
To answer the questions aforementioned, the present study adopted Chinese two-character words and pseudowords. To examine the familiarity-by-repetition interaction in duration judgment, Experiment 1 compared the repetition effect of Chinese words with that of pseudowords in a duration discrimination task. With our experimental design, if repeated pseudowords produce duration compression similar to that of repeated words, then the magnitude of the repetition compression in duration is not mediated by stimulus familiarity. On the other hand, should duration compression be stronger for repeated words than that of repeated pseudowords, we can argue that familiarity does interact with the effect. In Experiment 2 , we explore whether the apparent duration is compressed when the standard-comparison pair shares one constituent character and whether the constituent repetition effect is affected by familiarity. More specifically, in Experiment 2a, we administer four experimental conditions: whole-word repetition, first-character repetition, second-character repetition, and novel baseline. In Experiment $2 \mathrm{~b}$, we adopt the same design used in Experiment 2a and test the constituent repetition effect for pseudowords containing two unrelated characters. Note that we designed the first- and second-character repetition as two separate conditions to avoid inconsistent results regarding the interaction between constituent position and repetition effect as reported in previous studies (Zhou et al., 1999; Wu et al., 2016).

\section{EXPERIMENT 1}

\section{Methods \\ Participants}

Twenty students from Jiangnan University volunteered to take part in the experiment (six females; mean age 23.4 years). All participants were native speakers of Chinese, with normal or corrected-to-normal vision. All participants gave written informed consent before the experiment.

\section{Stimuli and Apparatus}

One hundred ten Chinese bimorphemic words and 110 pseudowords were selected to serve as the experimental stimuli. For words (e.g., 机器 /ji 1 qi 4/, meaning “machine"), the average frequency was 44 words/million, and the mean of strokes was 16. Pseudowords (mean of strokes: 17) were constructed by combining the existing characters to generate non-existing and non-interpretable bimorphemic stimuli (e.g., 提萌/ti 2 meng 2/, no meaning). Words/pseudowords were equally divided into two groups (word, Group $A_{w}$ and $B_{w}$; pseudoword, Group $\mathrm{A}_{\mathrm{p}}$ and $\left.\mathrm{B}_{\mathrm{p}}\right)$.

Words in Groups $A_{w}$ and $B_{w}$ were matched for word frequency and the number of strokes (both $p s>0.1$ ). Likewise, the number of strokes between the two groups of pseudowords was also matched $(p>0.1)$. The words from Group $A_{w}$ served as the standard stimulus, which could be followed by either an identical word or a different comparison word from Group $B_{w}$. The method used for pseudoword presentations was similar to the presentation of words. A 2 (repetition: repeated vs. novel) $\times 2$ (word type: word vs. pseudoword) repeated-measures design was used in the experiment. The words/pseudowords (about $2.9^{\circ} \times 2.3^{\circ}$ in size at the $57-\mathrm{cm}$ viewing distance) were presented on a 24 -in. LCD monitor with a refresh rate of $100 \mathrm{~Hz}$. All visual stimuli were displayed in white on a black background. The left- and right-arrow keyboard keys were used as response keys. Each participant performed the experiment individually in a dimly illuminated room. The experiment was programmed with Matlab using the Psychophysics Toolbox (Brainard, 1997).

\section{Procedure}

The experiment adopted a classic duration comparison task. An illustration of the stimulus presentation is shown in Figure 1. Each trial started with a fixation cross, presented for $800 \mathrm{~ms}$ in the center of the screen, thereafter replaced with a blank interval of 500-800 ms. In the sequence, the standard stimulus was displayed for a fixed duration of $600 \mathrm{~ms}$, followed by a 300-500 ms interstimulus interval (ISI). Next, the comparison stimulus was presented for one of the five possible durations $400,500,600,700$, or $800 \mathrm{~ms}$ - which was followed by a $500 \mathrm{~ms}$ blank interval. Finally, the onset of a question mark ("?") indicated that the participant should respond by pressing the left-arrow key if they perceived the duration of the comparison stimulus as being shorter than that of the standard stimulus, or the right-arrow key if they thought the comparison duration was longer. The intertrial interval (ITI) was set to 2,000 ms. All conditions were presented in random order, across 10 experimental blocks of 44 trials (22 times for each experimental condition). The words/pseudowords (each was used twice as the comparison stimulus) were randomly assigned to the comparison durations in each condition. The participants could take a break between blocks. Prior to the experimental session, each participant took part in a practice session of two blocks of 20 trials each. The test session lasted about $50 \mathrm{~min}$.

\section{Results}

We calculated the mean proportions of "longer" judgments (i.e., the comparison durations that were perceived as longer than the standard one) for each one of the five comparison durations, separately for each experimental condition and each participant. Thereafter, we fitted the psychometric curves to these proportions by using a logistic function. From the fitted curves, the point of subjective equality (PSE) and the just-noticeable difference (JND) were estimated. The PSE was the comparison duration at the $50 \%$ point of the curve as a measure of perceived duration (Treutwein and Strasburger, 1999; Birngruber et al., 2015; Jia and Shi, 2017). A lower PSE means longer perceived duration. The JND measure, as an index of discrimination sensitivity, was calculated by a half of the duration difference between the 25 and $75 \%$ points of the fitted response curves. A lower JND indicates better temporal discrimination. The unit of JND is represented in milliseconds.

The mean PSEs $( \pm$ SE) were $594( \pm 15), 552( \pm 12)$, $585( \pm 16)$, and $554( \pm 13) \mathrm{ms}$ for repeated-word ("Rw"), 


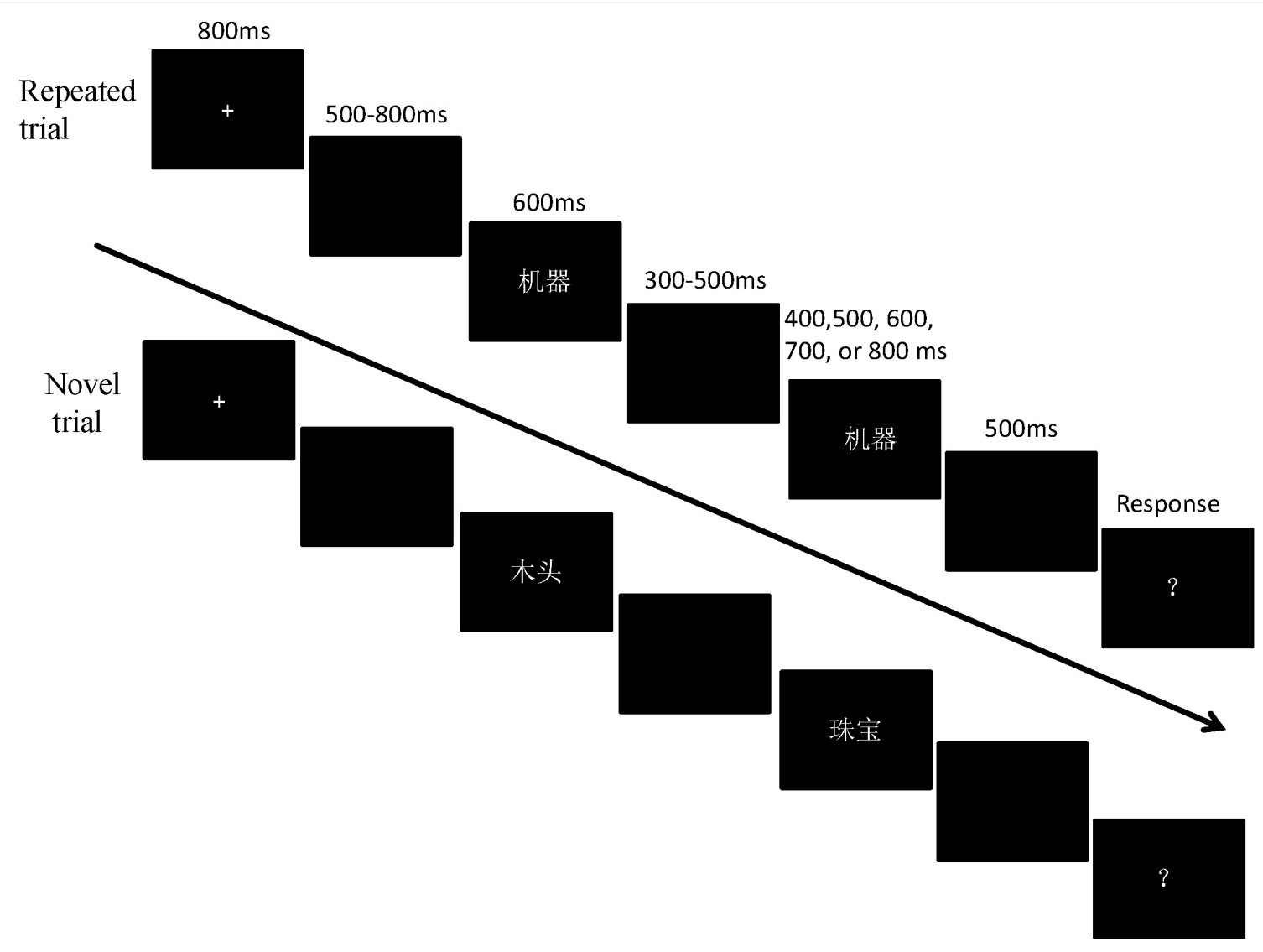

FIGURE 1 | Schematic illustrations of stimuli presentation in Experiment 1. In the repeated trials, the standard and comparison words/pseudowords were identical (e.g., 机器/ji 1 qi 4/, meaning “machine”). In novel trials, the standard and comparison words/pseudowords were different (e.g., the standard: 木头/mu 4 tou 1/, meaning "wood"; the comparison: 珠宝/zhu 1 bao 3/, meaning "jewelry").

novel-word ("Nw"), repeated-pseudoword ("Rp"), and novelpseudoword ("Np") conditions, respectively. Figure 2 shows the psychometric curves of the duration comparison task and the mean PSEs for all four experimental conditions. A repeated-measures ANOVA with the factors repetition (repeated vs. novel) and word type (word vs. pseudowords) was conducted on PSEs and JNDs. Regarding the analysis of the PSEs, the results revealed a significant main effect of repetition, $F(1,19)=5.40, p<0.05, \eta_{\mathrm{p}}{ }^{2}=0.22$. The higher PSEs observed in the repeated condition (with respect to the novel condition) indicate duration compression. Neither the main effect of word type, $F(1,19)=0.39, p=0.54$, $\eta_{\mathrm{p}}{ }^{2}=0.02$, nor their interaction, $F(1,19)=2.62, p=0.12$, $\eta_{\mathrm{p}}{ }^{2}=0.12$, reached statistical significance. As for the analysis of JNDs, however, no significant effect was found [repetition, $F(1,19)=0.87, p=0.36, \eta_{\mathrm{p}}{ }^{2}=0.04$; word type, $F(1$, $19)=1.19, p=0.29, \eta_{\mathrm{p}}{ }^{2}=0.06$; interaction, $F(1,19)=0.06$, $\left.p=0.81, \eta_{\mathrm{p}}{ }^{2}=0.003\right]$. The findings revealed in Experiment 1 showed that the repetition of words and pseudowords produces comparable duration compression, thus indicating that familiarity did not interact with the repetition duration effect. To further investigate whether familiarity mediates duration perception when a pair of stimuli share a common constituent character, Experiment 2 compares whole repetition, first-character repetition, and second-character repetition for words (Experiment 2a) and pseudowords (Experiment 2b).

\section{EXPERIMENT 2}

\section{Methods}

\section{Participants}

Thirty-nine students from Jiangnan University volunteered to take part in the experiments (20 in Experiment $2 \mathrm{a}$ and 19 in Experiment 2b; 24 females; mean age: 21.6 years). All participants were native speakers of Chinese, with normal or corrected-tonormal vision. Written informed consent was obtained from each participant before the experiments.

\section{Stimuli and Apparatus}

The experimental design, stimulus presentation, and apparatus were identical to those used in Experiment 1, except for the addition of the "constituent repetition" conditions (see below). Fifty-five bimorphemic words (e.g., 部落/bu 4 luo 4/, meaning "tribe") in Experiment 2a were selected to serve as standard stimuli, followed by one of four types of comparison 


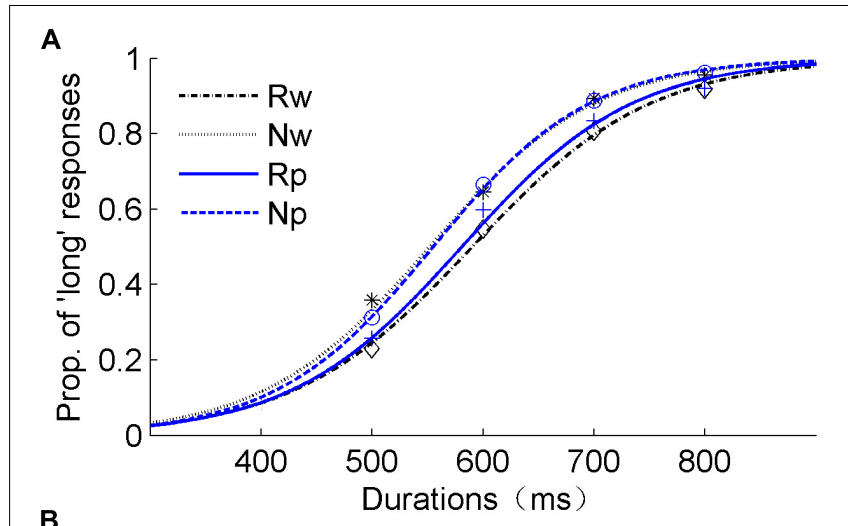

B

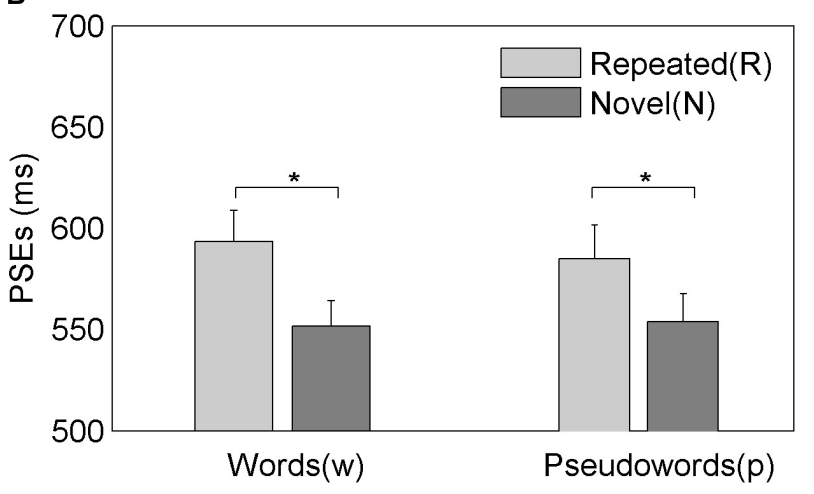

FIGURE 2 | Results of Experiment 1. (A) Mean proportions of "long" responses against the comparison durations plotted with fitted psychometric curves representing the Rw, Nw, Rp, and Np conditions. (B) The mean points of subjective equality (PSEs) and respective standard errors are shown for the four conditions. ${ }^{*} p<0.05$.

stimulus: (i) identical word (e.g., 部落), (ii) word with repetition of the constituent character at the first position (e.g., 部件/bu 4 jian 4/, meaning "component"), (iii) word with repetition of the constituent character at the second position (e.g., 角落/jiao 3 luo 4/, meaning “corner”), and (iv) novel word (e.g., 珠宝/zhu 1 bao 3/, meaning "jewelry"). For the standard-comparison pairs sharing one character, the repeated characters had the same phonology and orthography. The comparison words were matched in frequency (mean 48 words/million) and in number of strokes (mean 15) across the four conditions (all ps $>0.1$ ). The frequency and number of strokes between the shared characters in the first and second positions were also equal (both $p s>0.1$ ). For easy reference, we labeled the four conditions in Experiment $2 \mathrm{a}$ as (i) word repetition ("Wr"), (ii) first-character repetition ("FCr"), (iii) second-character repetition ("SCr"), and (iv) novel baseline ("Nb"). The experimental conditions of Experiment $2 b$ were identical to those of Experiment 2a, except that the stimuli used for duration judgment were pseudowords. Like Experiment 2a, the relevant conditions were matched in the number of strokes and character frequencies (all $p s>0.1$ ).

\section{Procedure}

The experimental procedure adopted in Experiment 2 was identical to that used in Experiment 1, except for the experimental conditions, that is, four experimental conditions under word category (Experiment 2a) and another four experimental conditions under the pseudoword category (Experiment 2b).

\section{Results}

Experiment 2a compared the influence of word repetition with constituent character repetition on duration judgment. The mean PSEs $( \pm S E)$ were $610( \pm 10), 561( \pm 11), 572( \pm 9)$, and $557( \pm 11) \mathrm{ms}$, for word repetition, first-character repetition, second-character repetition, and novel baseline conditions, respectively (Figure 3). A one-way repeated-measures ANOVA with Greenhouse-Geisser correction for PSEs revealed a significant main effect of repetition, $F(1.936,36.780)=10.64$, $p<0.001, \eta_{\mathrm{p}}{ }^{2}=0.36$. Further pairwise comparisons with Holm-Bonferroni correction revealed that mean PSEs were significantly higher for the word repetition condition than the first-character repetition (mean differences: $49 \mathrm{~ms}$, corrected $p=0.006$ ), the second-character repetition (mean differences: $38 \mathrm{~ms}$, corrected $p=0.024$ ), and the novel baseline conditions (mean differences: $53 \mathrm{~ms}$, corrected $p=0.006$ ). The analysis failed to reveal any significant difference of PSEs between the first-character repetition and second-character repetition (corrected $p=0.212$ ), between the second-character repetition

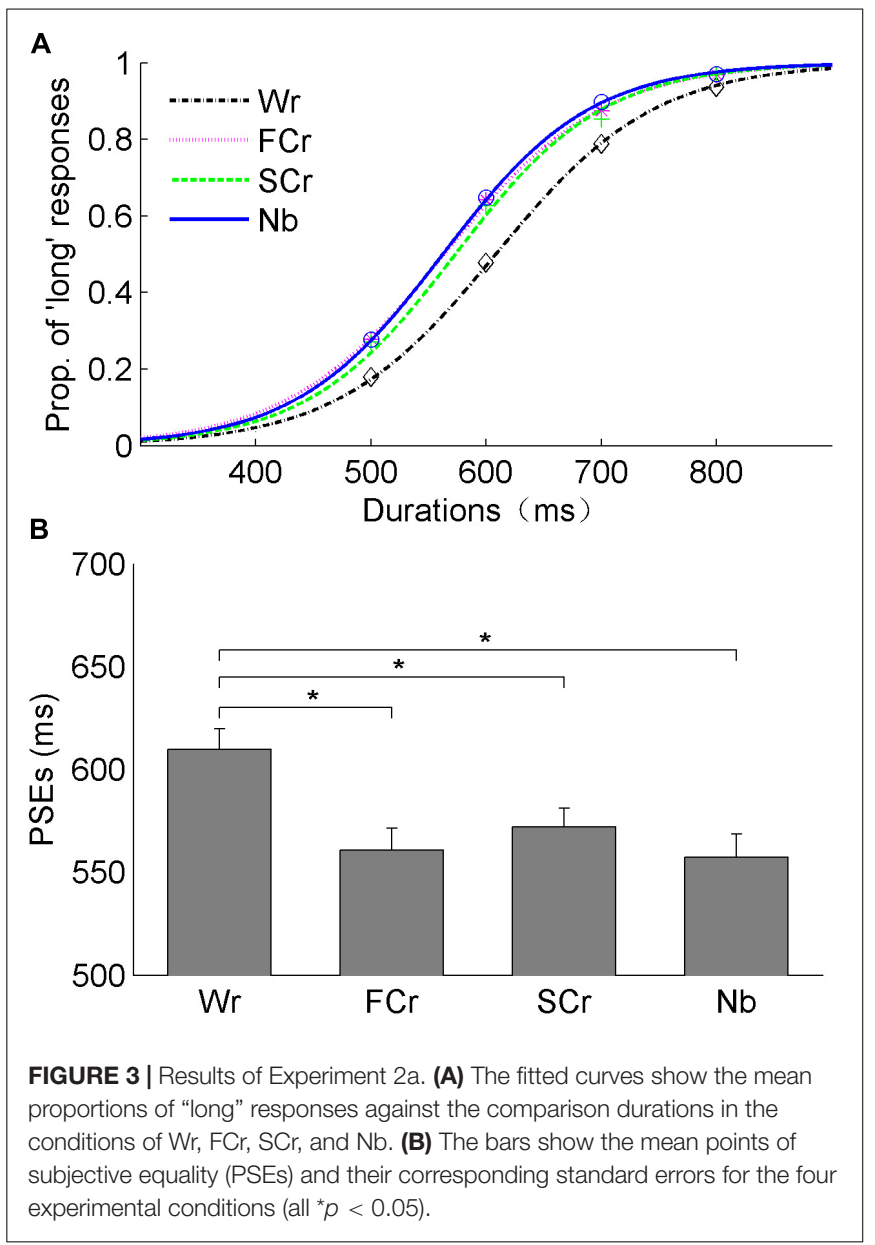




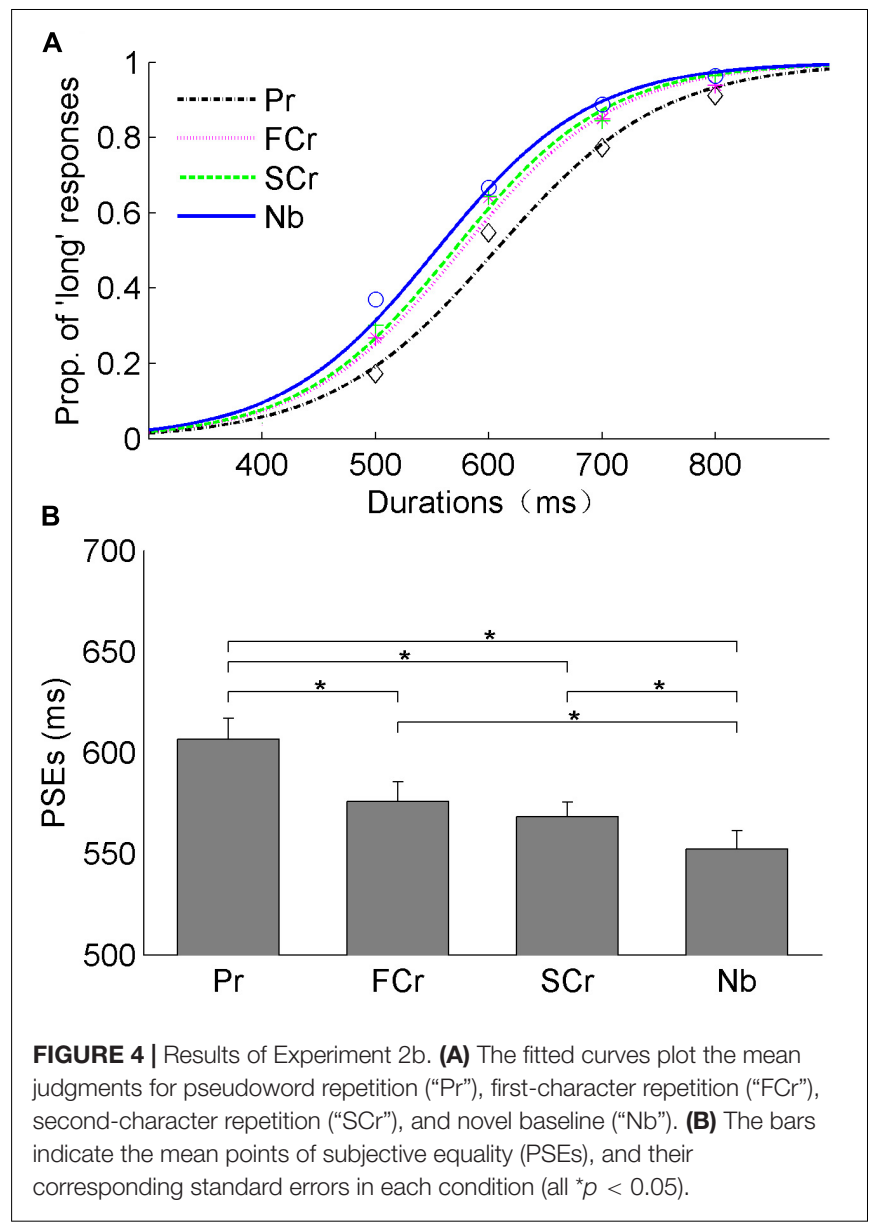

and novel baseline (corrected $p=0.189$ ), and between the firstcharacter repetition and novel baseline (corrected $p=0.676$ ). We applied the same statistical methods to analyze the JNDs across the experimental conditions; nonetheless, the results failed to show any statistically significant effect, Greenhouse-Geisser correction, $F(1.957,37.175)=0.28, p=0.84, \eta_{\mathrm{p}}{ }^{2}=0.015$.

As for Experiment $2 \mathrm{~b}$ comparing the influence of pseudoword repetition with constituent character repetition on duration judgment, the mean PSEs $( \pm S E)$ were $607( \pm 10), 576( \pm 9)$, $568( \pm 7)$, and $552( \pm 9) \mathrm{ms}$ for pseudoword repetition, firstcharacter repetition, second-character repetition, and novel baseline conditions, respectively (Figure 4). The ANOVA revealed a significant difference between the four experimental conditions, $F(3,54)=16.16, p<0.001, \eta_{\mathrm{p}}{ }^{2}=0.47$. The posthoc Holm-Bonferroni correction showed that mean PSEs were significantly higher for the pseudoword repetition condition as compared with the first-character repetition (mean differences: $31 \mathrm{~ms}$, corrected $p=0.008$ ), second-character repetition (mean differences: $39 \mathrm{~ms}$, corrected $p=0.000$ ), and novel baseline conditions (mean differences: $55 \mathrm{~ms}$, corrected $p=0.000$ ). Both PSEs for first-character repetition (mean differences: $24 \mathrm{~ms}$, corrected $p=0.015$ ) and for second-character repetition (mean differences: $16 \mathrm{~ms}$, corrected $p=0.034$ ) were significantly higher than the novel baseline. No significant difference in PSEs between the first- and second-character repetitions was found (mean differences: $8 \mathrm{~ms}$, corrected $p=0.224)$. Last but not least, repetition conditions did not influence JNDs, $F(3,54)=0.57$, $p=0.64, \eta_{\mathrm{p}}^{2}=0.03$.

In short, our statistical analysis revealed a different pattern of results in Experiments $2 \mathrm{a}$ and $2 \mathrm{~b}$. That is, whereas in Experiment 2a (i.e., with word stimuli) neither first- nor secondcharacter repetition conditions induced duration compression relative to novel baseline, the opposite was true for Experiment $2 b$ (i.e., with pseudoword stimuli). More precisely, relative to novel baseline, pseudowords with both the first- and second-character repetition induced duration compression.

\section{DISCUSSION}

The current study investigated whether stimulus familiarity interacted with the repetition effect observed in a duration discrimination task using words and pseudowords. In Experiment 1, we observed that the repetition of Chinese words and pseudowords generated comparable duration compressions. In Experiment 2, we examined the constituent repetition effect when the standard-comparison stimuli shared the first or second constituent character. Interestingly, we did not find the constituent repetition effect for words (Experiment 2a). However, we did find a duration compression effect for constituent character repetition in pseudowords, although the strength of the compression was smaller than that of whole pseudoword repetition (Experiment $2 b$ ). We take these findings to imply that, (i) according to Experiment 1, familiarity alone does not impact the duration compression caused by the whole stimulus repetition, as words and pseudowords produced comparable duration compression. However, (ii) familiarity does mediate the effect of constituent character repetition in duration judgment as observed in Experiment 2.

The present study provides clear evidence in support of the repetition duration compression for both words and pseudowords, thus joining a stream of recent reports of the effect on duration judgment tasks (Birngruber et al., 2015; Cai et al., 2015; Matthews and Gheorghiu, 2016; Jia and Shi, 2017). Further, our study extends the current literature by demonstrating that duration compression is virtually comparable between words and pseudowords. Therefore, our findings indicate that a single repetition of an unfamiliar stimulus is sufficient to induce duration compression with a magnitude equivalent to that of a familiar stimulus. More specifically, the effect on duration estimation following the repetition of a stimulus is not proportionally influenced by the familiarity that the observer may have with it.

What is more, the new findings presented point to a different direction from those studies that employed lexical decision tasks, where greater repetition facilitation for words relative to pseudowords was observed (Henson et al., 2000; Fiebach et al., 2005; Orfanidou et al., 2006). The divergence between the current and previous studies is likely due to the different levels of processing specific to the requirements of each task. For instance, whereas lexical-related processing is essential in a lexical decision task, semantic processing seems not important in a duration 
judgment task. As an illustration, Jia and Shi (2017) showed in a recent study that orthographic and phonological information was more important for the repetition duration effect than semantic information. In their study, only phonological repetition between Chinese characters induced duration compression, whereas semantic repetition failed to induce the compression effect.

Concerning the effect of constituent character repetition in duration judgment when the standard-comparison pair shared a common character, we found disparate results for words and pseudowords. The perceived duration of the comparison pseudowords with an identical first or second character to that of the standard pseudoword was compressed relative to novel baseline, whereas the presentation of word pairs sharing constituent characters did not show this effect. The present findings suggest that familiarity modulates the effect of constituent repetition in the perception of time at least with Chinese words. This is contrary to studies that administered lexical decision or identification tasks, wherein the effect of constituent repetition for word was shown when the standard and comparison stimuli shared constituent characters (Zhou et al., 1999; Tsang et al., 2014; Wu et al., 2016). A possible explanation for this disparity stems from the idea that semanticrelated judgment of words, such as lexical decision, requires a deeper level of processing because each constituent morpheme must be interpreted. In a duration judgment task, however, lexical interpretation is "overwritten" by time estimation as the focus of the task is on how long a stimulus is present on the screen rather than on its semantic processing. Over and above that, words are composed of constituent characters with a familiar linkage, leading to a more effective performance when words are processed holistically, as compared with when they are processed at the morpheme level. On this note, it is possible that when the standard and comparison words share only a constituent character in a duration judgment task, the latter is processed as a novel stimulus. Conversely, as the structure of pseudowords is formed by unrelated characters, it is likely that each stimulus is processed at the character level. This idea is supported by the findings that extensive prior exposure to a stimulus enhanced the associations among its features compared with exposure to an untrained stimulus (Meyer and Olson, 2011; Grotheer and Kovács, 2014). In other words, familiar stimuli (e.g., words) tend to be processed at an integrated level, whereas unfamiliar stimuli (e.g., pseudowords) are processed at the component level.

In order to explain the repetition duration effect, the attentional account assumes that unexpected stimuli capture more attention than do expected stimuli, thus resulting in longer subjective duration (Tse et al., 2004; Matthews and Gheorghiu, 2016; Matthews and Meck, 2016). In this specific regard, the attentional account supports our results as we found that the duration of novel words/pseudowords were judged as being longer than that of repeated stimuli (Tse et al., 2004). However, the attentional account does not explain the fact that the influence of constituent repetition led to different outcomes in the word and pseudoword conditions. Moreover, as per the attentionalgate model, when more attentional resources are given to the non-temporal features of an unexpected stimulus, less attention is devoted to the temporal features. This process may skip the temporal pulses otherwise accounted for, thus shortening the subjective duration of the unexpected stimulus (Zakay and Block, 1997; Ulrich and Bausenhart, 2019). This outcome is the opposite effect commonly observed in the classic duration compression of repeated stimuli. Similarly, the arousal account also fails to offer a satisfactory explanation for the divergent findings regarding constituent repetition of words and pseudowords. In other words, JNDs indicating the sensitivity to temporal discrimination did not show significant changes among different experimental conditions. Therefore, the current results cannot be thoroughly explained by either attentional or arousal accounts.

A further, possible explanation for the current results concerns the influence of memory and decision under the framework of the internal clock (Gibbon, 1991; Wearden, 1992). For instance, memory-related factors such as the ISI between the standard and comparison stimuli have been shown to affect the repetition effect, as demonstrated in a study by Matthews (2015) where the repetition effect in the short (e.g., $306 \mathrm{~ms}$ ) and long (e.g., $2,000 \mathrm{~ms}$ ) ISI conditions were compared. The results showed that the repetition effect only occurred in the short-ISI condition, indicating that the effect was short-lived. Consequently, it is unlikely that memory-related factors influenced the present findings, as short ISIs (300-500 ms) were used across all experimental conditions. In the same vein, it is improbable that the present results reflect a decision bias. This is because the repetition duration effect has been manifested in other judgment tasks such as the equality judgment task, which is not affected by response bias (Birngruber et al., 2014). Over and above that, there is a consensus that the repetition duration effect is rather a response to perceptual bias (Birngruber et al., 2014; Matthews and Gheorghiu, 2016; Ulrich and Bausenhart, 2019).

An alternative to the internal clock mechanism, namely, the repetition suppression account, argues that neural responses to repeated words/pseudowords get suppressed, which then leads to a reduced perceived duration (Grill-Spector et al., 2006; Noguchi and Kakigi, 2006; Eagleman and Pariyadath, 2009) where the size of the repetition duration compression is relative to the difference between the comparison and standard stimuli. Although this view might explain the differential magnitudes of duration compression between whole-repeated and constituentrepeated pseudowords, it does not explain the lack of duration compression for constituent repeated words. Indeed, some neuroimaging studies have shown that neural responses are not only suppressed but also enhanced for repetition (Segaert et al., 2013), indicating that the neural responses do not always reflect the perceived duration.

Last but not least, the predictive coding account suggests that the expected stimulus produces a shortening of subjective duration to optimize coding efficiency, a process within which the magnitude of the repetition duration effect depends on the similarity between the standard and comparison stimuli (Rao and Ballard, 1999; Friston, 2005). Specifically, the fewer the prediction errors (from the difference between the predicted and actual signals),the shorter the subjective duration perceived. Applied to the present study, we observed a discrepancy between the standard stimulus and two comparison conditions, namely, the constituent repeated and novel stimulus. Thus, prediction errors 
differed among three experimental conditions: no prediction error for whole-repeated stimuli, partial prediction error for the constituent repeated stimuli, and the most prediction errors for novel stimuli. In other words, the subjective duration of whole-repeated stimuli was perceived as being shorter than the other two stimuli, whereas the presentation of the constituent repeated stimulus was perceived as shorter than the novel, in the pseudoword condition. However, the constituent repetition of words did not show a duration compression. We suggest that the implicit expectation may encompass words at a configural and/or holistic level in duration judgment. As a result, word pairs sharing one constituent character evoke prediction errors similar to that evoked during the judgment of novel word pairs. On the contrary, when participants see the standard pseudoword with no relation between constituent characters, naturally, they expect an unfamiliar comparison stimulus with similar characteristics. However, when standard and comparison pseudowords share a constituent character, the surprise prediction error is only partial. Consequently, the compression effect for pseudowords is reduced compared with whole words. Consequently, this process places the predictive coding account as a more comprehensive explanation for the present repetition duration compression than the aforementioned accounts of attentional/arousal and repetition suppression (Jia and Shi, 2017).

In summary, the present study provides clear evidence that the repetition of words and pseudowords promotes analogous duration compression, indicating that familiarity per se does not interact with the effect of the whole repetition in duration judgment. Moreover, we revealed that when the standard and comparison stimuli shared a constituent character at the first or second position, there was a repetition compression for pseudowords; however, it was reduced in relation to the whole pseudoword repetition. By contrast, the constituent repetition of words did not show the compression effect, as words with only a constituent repetition might have been processed as a novel word altogether. We suggest this outcome indicates that pseudowords are processed at the component level whereas words are processed holistically in a duration judgment task.

\section{REFERENCES}

Birngruber, T., Schroter, H., and Ulrich, R. (2014). Duration perception of visual and auditory oddball stimuli: does judgment task modulate the temporal oddball effect? Atten. Percept. Psychophys. 76, 814-828. doi: 10.3758/s13414013-0602-2

Birngruber, T., Schröter, H., and Ulrich, R. (2015). The influence of stimulus repetition on duration judgments with simple stimuli. Front. Psychol. 6:1213. doi: $10.3389 /$ fpsyg.2015.01213

Brainard, D. (1997). The psychophysics toolbox. Spat. Vis. 10, 433-436. doi: 10. 1163/156856897X00357

Cai, M., Eagleman, D. M., and Ma, W. (2015). Perceived duration is reduced by repetition but not by high-level expectation. J. Vis. 15:19. doi: 10.1167/15.13.19

Ciria, A., Lopez, F., and Lara, B. (2019). Perceived duration: the interplay of topdown attention and task-relevant information. Front. Psychol. 10:490. doi: 10. 3389/fpsyg.2019.00490

De Baene, W., and Vogels, R. (2010). Effects of adaptation on the stimulus selectivity of macaque inferior temporal spiking activity and local field potentials. Cereb. Cortex 20, 2145-2165. doi: 10.1093/cercor/bhp277
Therefore, we claim that familiarity interacts with the constituent repetition effect, but differently for words and pseudowords. Thus, the pattern of results across the two experiments favors the idea that the magnitude of the repetition compression depends on the difference between the actual signal and prediction.

\section{DATA AVAILABILITY STATEMENT}

The datasets generated for this study are available on request to the corresponding authors.

\section{ETHICS STATEMENT}

The studies involving human participants were reviewed and approved by the Ethics Committee of Jiangnan University. The patients/participants provided their written informed consent to participate in this study. Written informed consent was obtained from the individual(s) for the publication of any potentially identifiable images or data included in this article.

\section{AUTHOR CONTRIBUTIONS}

LJ, XZ, and XW developed the design of the study. CD collected the data. LJ, XZ, and LW performed the data analyses. LJ, XZ, LW, and XW contributed to the writing and drafting the manuscript.

\section{FUNDING}

This study was supported by the Nature Science Foundation of Jiangsu Province in China (Grant Nos. BK20160171 to LJ and BK20191485 to LW) and National Natural Science Foundation of China (Grant No. 31600876 to XZ). The funding institution had no role in the study design, data collection, analysis decision to publish, or preparation of the manuscript.

Desimone, R. (1996). Neural mechanisms for visual memory and their role in attention. Proc. Natl. Acad. Sci. U.S.A. 93, 13494-13499. doi: 10.1073/pnas.93. 24.13494

Eagleman, D. M., and Pariyadath, V. (2009). Is subjective duration a signature of coding efficiency? Philos. Trans. R. Soc. Lond. B. Biol. Sci. 364, 1841-1851. doi: 10.1098/rstb.2009.0026

Fiebach, C. J., Gruber, T., and Supp, G. G. (2005). Neuronal mechanisms of repetition priming in occipitotemporal cortex: spatiotemporal evidence from functional magnetic resonance imaging and electroencephalography. J. Neurosci. 25, 3414-3422. doi: 10.1523/jneurosci.4107-04.2005

Friston, K. (2005). A theory of cortical responses. Philos. Trans. R. Soc. Lond. 360, 815-836. doi: 10.1098/rstb.2005.1622

Gibbon, J. (1991). Origins of scalar timing. Learn. Motiv. 22, 3-38. doi: 10.1016/ 0023-9690(91)90015-Z

Grill-Spector, K., Henson, R., and Martin, A. (2006). Repetition and the brain: neural models of stimulus-specific effects. Trends Cogn. Sci. 10, 14-23. doi: 10.1016/j.tics.2005.11.006

Grotheer, M., and Kovács, G. (2014). Repetition probability effects depend on prior experiences. J. Neurosci. 34, 6640-6646. doi: 10.1523/jneurosci.5326-13.2014 
Henson, R., Shallice, T., and Dolan, R. (2000). Neuroimaging evidence for dissociable forms of repetition priming. Science 287, 1269-1272. doi: 10.1126/ science.287.5456.1269

Henson, R. N. A., and Rugg, M. D. (2003). Neural response suppression, haemodynamic repetition effects, and behavioural priming. Neuropsychologia 41, 263-270. doi: 10.1016/S0028-3932(02)00159-8

Jia, L., and Shi, Z. (2017). Duration compression induced by visual and phonological repetition of Chinese characters. Atten. Percept. Psychophys. 79, 2224-2232. doi: 10.3758/s13414-017-1360-3

Matthews, W., Terhune, D., Rijn, H., Eagleman, D., Sommer, M., and Meck, W. (2014). Subjective duration as a signature of coding efficiency: emerging links among stimulus repetition. Predictive Coding, and Cortical GABA Levels. Timing Time Percept. Rev. 1, 1-11. doi: 10.1163/24054496-00101005

Matthews, W. J. (2011). Stimulus repetition and the perception of time: the effects of prior exposure on temporal discrimination, judgment, and production. PLoS One 6:e19815. doi: 10.1371/journal.pone.0019815

Matthews, W. J. (2015). Time perception: the surprising effects of surprising stimuli. J. Exp. Psychol. Gen. 144, 172-197. doi: 10.1037/xge0000041

Matthews, W. J., and Gheorghiu, A. I. (2016). Repetition, expectation, and the perception of time. Curr. Opin. Psychiatr. 8, 110-116. doi: 10.1016/j.cobeha. 2016.02.019

Matthews, W. J., and Meck, W. H. (2016). Temporal cognition: connecting subjective duration to perception, attention and memory. Psychol. Bull. 142, 865-907. doi: 10.1037/bul0000045

Meyer, T., and Olson, C. R. (2011). Statistical learning of visual transitions in monkey inferotemporal cortex. Proc. Natl. Acad. Sci. U.S.A. 108, 19401-19406. doi: 10.1073/pnas.1112895108

Noguchi, Y., and Kakigi, R. (2006). Time representations can be made from nontemporal information in the brain: an MEG study. Cereb. Cortex 16, 1797-1808. doi: 10.1093/cercor/bhj117

Orfanidou, E., Marslen-Wilson, W. D., and Davis, M. H. (2006). Neural response suppression predicts repetition priming of spoken words and pseudowords. J. Cogn. Neurosci. 18, 1237-1252. doi: 10.1162/jocn.2006.18.8.1237

Pariyadath, V., and Eagleman, D. M. (2012). Subjective duration distortions mirror neural repetition suppression. PLoS One 7:e49362. doi: 10.1371/journal.pone. 0049362

Rao, R. P. N., and Ballard, D. H. (1999). Predictive coding in the visual cortex: a functional interpretation of some extra-classical receptive-field effects. Nat. Neurosci. 2, 79-87. doi: 10.1038/4580

Saurels, B., Lipp, O., Yarrow, K., and Arnold, D. (2019). Predictable events elicit less visual and temporal information uptake in an oddball paradigm. Atten. Percept. Psychophys. doi: 10.3758/s13414-019-01899-x

Schindel, R., Rowlands, J., and Arnold, D. H. (2011). The oddball effect: perceived duration and predictive coding. J. Vis. 11:17. doi: 10.1167/11.2.17

Segaert, K., Weber, K., de Lange, F. P., Petersson, K. M., and Hagoort, P. (2013). The suppression of repetition enhancement: a review of fMRI studies. Neuropsychologia 51, 59-66. doi: 10.1016/j.neuropsychologia.2012.11.006
Skylark, W. J., and Gheorghiu, A. I. (2017). Further evidence that the effects of repetition on subjective time depend on repetition probability. Front. Psychol. 8:1915. doi: 10.3389/fpsyg.2017.01915

Tenpenny, P. L. (1995). Abstractionist versus episodic theories of repetition priming and word identification. Psychon. Bull. Rev. 2, 339-363. doi: 10.3758/ bf03210972

Treisman, M. (1963). Temporal discrimination and the indifference interval: implications for a model of the 'internal clock'. Psychol. Monogr. 77, 1-31. doi: $10.1037 /$ h0093864

Treutwein, B., and Strasburger, H. (1999). Fitting the psychometric function. Percept. Psychophys. 61, 87-106. doi: 10.3758/BF03211951

Tsang, Y. K., Wong, A. W.-K., Huang, J., and Chen, H.-C. (2014). Morphoorthographic and morpho-semantic processing in word recognition and production: evidence from ambiguous morphemes. Lang. Cogn. Neurosci. 543560. doi: 10.1080/01690965.2013.790554

Tse, P. U., Intriligator, J., Rivest, J., and Cavanagh, P. (2004). Attention and the subjective expansion of time. Percept. Psychophys. 66, 1171-1189. doi: 10.3758/ BF03196844

Ulrich, R., and Bausenhart, K. (2019). "The temporal oddball effect and related phenomena: cognitive mechanisms and experimental approaches," in The Illusions of Time: Philosophical and Psychological Essays on Timing and Time Perception, eds V. Arstila, A. Bardon, S. E. Power, and A. Vatakis (Cham: Springer Nature Switzerland), 71-89. doi: 10.1007/978-3-030-22048-8_5

Ulrich, R., Nitschke, J., and Rammsayer, T. (2006). Perceived duration of expected and unexpected stimuli. Psychol. Res. 70, 77-87. doi: 10.1007/s00426-004-0195-4

Wearden, J. H. (1992). Temporal generalization in humans. J. Exp. Psychol. Anim. B 18, 134-144. doi: 10.1037/0097-7403.18.2.134

Wu, Y., Tsang, Y. K., Wong, W. K., and Chen, H. C. (2016). The processing of homographic morphemes in Chinese: an ERP study. Lang. Cogn. Neurosci. 32, 1-15. doi: 10.1080/23273798.2016.1227857

Zakay, D., and Block, R. A. (1997). Temporal cognition. Curr. Dir. Psychol. Sci. 6, 12-16. doi: 10.1111/1467-8721.ep11512604

Zhou, X., Marslen-Wilson, W., Taft, M., and Shu, H. (1999). Morphology, orthography, and phonology in reading Chinese compound words. Lang. Cogn. Process. 14, 525-565. doi: 10.1080/016909699386185

Conflict of Interest: The authors declare that the research was conducted in the absence of any commercial or financial relationships that could be construed as a potential conflict of interest.

Copyright (c) 2020 Jia, Deng, Wang, Zang and Wang. This is an open-access article distributed under the terms of the Creative Commons Attribution License (CC BY). The use, distribution or reproduction in other forums is permitted, provided the original author(s) and the copyright owner(s) are credited and that the original publication in this journal is cited, in accordance with accepted academic practice. No use, distribution or reproduction is permitted which does not comply with these terms. 